\title{
SDS: A Framework for Secure Data Sharing in Database as a Service
}

\author{
R. Muthuram and Dr.G. Kousalya
}

\begin{abstract}
Cloud computing is a magic and attractive technology to the single user to large enterprises to store their data. It has less expensive and useful model for enterprise who concentrates more on themes instead of buying products. But, obstacle for the cloud is privacy and unauthorized access of users from internal or external. To achieve the privacy of data from the internal the client stores their sensitive data in the encrypted form. But for the large organizations, the delegated contents are shared by various users. So, we are in the need of an efficient access control mechanisms which coupled with encrypted data to privacy preserving data sharing among the users. In this paper, we present a novel approach to flexible data access control with re-encryption performed by the service provider. The basic design of this process is applying the two-level encryption on the data with access control. The service provider performs the second level encryption with user's proxy key which is unique to the users. This approach provides the selective authorization on the encrypted data and also provides the flexible access control. Even though it has lot of proxy keys as much of users, no need of key distribution. And also, the user should remember only his secret key. In the end of this paper, we analyze and resolve the solutions for the possible attacks.
\end{abstract}

Keywords--- Integrity, Access Control, Data Security, Re-encryption, DaaS.

\section{INTRODUCTION}

$\mathrm{N}$ OWADAYS, all the academic, scientific and business organizations hold a lot of data information for their applications and volume is increasingly very quickly. Those databases contain a critical concentration of sensitive information. To efficient data management, the organizations need to purchase necessary hardware and database products that products should deploy in the local system [3]. The organization also hires the database professionals to administrate the database and manage the network connectivity. This traditional solution has been getting increasingly expensive and impractical as the database systems and problems become larger and more complicated.

In such a scenario, database outsourcing [1] is becoming increasingly popular. A client's database is stored at an external database service provider. The main advantage of outsourcing is related to the costs of in-house versus

R. Muthuram, Assistant Professor, Alagappa Chettiar College of Engineering and Technology, Karaikudi.E-mail:muthuramrr@gmail.com

Dr.G. Kousalya, Professor, Coimbatore Institute of Technology, Coimbatore.E-mail:kousir@gmail.com

DOI:10.9756/BIJDM.8306 outsourced hosting. Moreover, the data owner can concentrate more attention on his core business. But, a significance of this trend towards outsourcing, the data owners lost their control on access control \& privacy of sensitive data. To obtain the privacy, the given data is encrypted and stored.

In this paper, we present a dynamic solution which provides the flexible access control management adopted by service provider by combining the re-encryption mechanism with access control policy of data owner. In this approach, the data contents of owner are encrypted with the owner's private data key and stored in the service provider. For each user, semi trusted party generates the different re-encryption key [4, 14] that delegated to the service provider. It allows the service provider to re-encrypt the cipher-texts using the corresponding re-encryption key of the legitimate user. The re-cipher texts are decrypted only with the legitimate user's private key. This approach effectively implements the selective authorization and dynamic policy updating

\section{RELATED WORK}

Shard Mehrotra proposed a new concept database as a service [1, 12, 13] in 2002 and proposed architecture NetDB2 model for the database service in internet. In this, data privacy is achieved by software and hardware level encryption [5]. In these encryption schemes, query evaluation time can be reduced by rewriting the queries. Rakesh Agarwal proposes the flexible data query approach [2] for the encrypted database. They proposed OPES architecture that allows the queries with comparison operators to be directly applied for encrypted database.

Other than the privacy of the data, integrity and authentication also should be ensured in the database as a service scenario. Narasimha et al. proposed a 'signature aggregation' technique [16] for providing efficient integrity in outsourced data model. They designed 'condensed RSA' scheme which performs well in unified client as well as multi queries models. In order to perform query execution efficiently, valentina proposed approach that combines the data fragmentation [6] with the encryption. Recently, they have given importance for secure hardware [18] componenttrust hardware and its application in the entrusted context. In [7], authors have proposed the technique of public key based homomorphic linear authenticator, which enables TPA to perform the auditing without demanding the local copy of data

Access control is another important problem in the database as a service for implementing data privacy. Even lot of access control mechanisms are available, there is no research for the issue of access control in DaaS scenario 
specifically till 2007. Damini has proposed a solution for access control management [8] by exploiting selective data encryption. The basic idea is to use different encryption keys for different data. To access such encrypted data, users have to decrypt them by using appropriate key. They propose a "key derivation" method that given a key and a piece of information publicly available, derives another key in the system. Gerome Miklau proposes a framework for enforcing access control policies on XML documents [15] using encryption. In this, the owner enforces the partial data encryption which describes access control policies. Recently, Anna Zych describes the key management method [19] for cryptographically enforced database. They proposed new algorithm to transforms an arbitrary access control matrix into user hierarchy tree to which public, private key pairs are assigned based on Diffie Helman Key generation scheme.

Bertino et al., [17] proposed how to guarantee the privacy of delegated policy. They proposed a scheme for selective distribution of context, which encoded as documents which are delivered through novel key management scheme. Users are able to reconstruct the keys to decrypt the authorized portions of document based on information received from document publisher. This system efficiently handles joining and leaving of subscribers with guaranteed security. Even though, a lot of access control schemes are described above, all the methods are exploit the same selective encryption method to implement access control based on partial relationship of the users of the system.

\section{SYSTEM MODEL}

In this section, we discuss about the architecture of proposed system. Access control based encryption system framework is shown in figure 3.1. From the framework, the system model has four entities: Data Owner, Key Distribution Center, Cloud service Provider, and Data Requester. Here, we discuss the four components and its functionalities.

\section{Components of System Model}

The architecture of proposed system model is shown above. This proposed model is used to share the user's data in flexible and efficient manner. The system model has four components. The functionality of each component is described as follows:

\section{A. Data Owner}

This is the basic component for the whole model. Its functionalities are as follows:

- Collect the Key pair from Certificate Key authority

- Creates the access control list and proxy key list and exported to the service provider

- Encrypt the file and generate the integrity and uploaded into service provider.

It has following subcomponents:

\section{Browser}

The browser is a small software tool that used to collect the key pairs. It sends the request to Certificate Key Authority to collect the Public/private key pair and symmetric key pair.
It also sends the identity to create the proxy key that collected in proxy key list.

\section{Encryption and Integrity}

The data owner stores their information in the untrusted service provider. To improve the trust on service provider, the owners will trans-code their data into another format. The owner uses either symmetric or asymmetric encryption algorithm.

\section{B. Data Service Provider}

This is the third party component that used to stores the user's data. It is untrusted component. It provides the database as a service. It also stores the access control lists and proxy key lists. The functionality is described as follows:

- It checks identity of the user who is authentic or not.

- If the user is authentic, then it checks that user can access the file or not.

- It performs the re-encryption and sends the re-encrypted data to the requester.

\section{Certificate Key Authority}

This is the third party component to generate the certificate and key for the user. It has Key generation algorithm to generate the private and public key pair or symmetric key which depends on the user requests. It also generates the Proxy key for the user who needs to access the data in data service provider. It also provides the certificate for authentication.

\section{Data Requester}

This is the component that is willing to access the information of the data owner. The functionalities of this component are described as follows:

- It sends their identity and collects the proxy key.

- It performs the decryption to know the plain text.

\section{FRAMEWORK MECHANISM}

The access control based encryption framework achieves flexible access control enforcement and selective authorization through second level encryption. This framework guarantees the integrity of the user data. Because of second level encryption, the service provider protects the data from the man in middle attack. This framework has seven algorithms.

Setup $\left(1^{\mathrm{k}}\right)$ : This algorithm is executed by the trusted Key authority KDC. It gets the security parameters from the data owner and generates the Master Secret Key and Master Public Key.

Key Generation (SK, PK): This algorithm performed by the KDC and generates one public and private key pair. This key pair is used to either protect the owner's data and also to verify the integrity.

Encryption (Key pair, Enc_alg): This is executed by the data owner. Enc_alg is either symmetric key algorithm or asymmetric algorithm. Like that, for all the files may applies the same key or separate key for each file. It generates ciphertext $\mathrm{CP}$ as output. 
Hash_MAC (CP, sk): It will be executed by the data owner. It gets cipher text $\mathrm{CP}$ as input and generates the irreversible hash code and that will be attached with the CP.

Re-Encrypt_Key_Generation (user_id): This algorithm performed by the KDC. It gets the user_id and generates the re-encrypt_key to perform the second level encryption. That key will be updated in the access control table.

Re-encryption (CP, rk): This algorithm is executed by the service provider. The user sends the request to view the owner file, and then the SP collects the encrypted file and applies the symmetric key encryption using user's re-encryption file. The re-encrypted file will sends to the requested user.

Decrypt_Verify (RCP, rk): This is performed by requested user. It gets the re-encrypted file as input and generates the plain text as output. It also verifies the integrity of the data.

In this framework, we are applying two level encryption mechanisms to the owner's data to avoid and protect the data from man in the middle attack. The first level encryption is performed by the data owner and the second level encryption or re-encryption is performed by the service provider. The first level encryption is performed either by public key approach or symmetric key approach.

\section{A. First Level Encryption}

This level encryption is performed by the data owner who collects the key pair from the KDC. This algorithm is performed by two different approaches.1. Public key approach and 2.Multi key approach.

\section{a. Public Key Approach}

In this approach, the data owner applies the proxy encryption mechanism directly. In this, the data owner uses the asymmetric encryption algorithm to encode the data. The data owner receives his public key and private key from the key authority through key generation algorithm. Then, the content of data owner is encrypted using his public key. At the same time, the private key which is used to decrypt the data is transferred to the user who requesting this content through secure channel. For e.g., consider the data owner's public key is $\mathrm{PK}_{\mathrm{DO}}$ and his plain text is $\mathrm{F}$. Then, the owner applies the encryption algorithm for the file content.

$$
\text { DO: } \mathrm{E}\left(\mathrm{F}, \mathrm{PK}_{\mathrm{DO}}\right) \rightarrow \mathrm{C}_{\mathrm{F}}
$$

Now, the data owner receives the one secret key from the key authority and applies Hash_MAC algorithm and generates the irreversible hash code. The file's hash code secret key is encrypted with public key of the owner.

$$
\begin{gathered}
\text { DO: } \mathrm{H} \_\mathrm{MAC}\left(\mathrm{Sk}_{\mathrm{F}}, \mathrm{C}_{\mathrm{F}}\right) \rightarrow \mathrm{H}_{\mathrm{F}} \\
\text { DO: } \mathrm{E}\left(\mathrm{Sk}_{\mathrm{F}}, \mathrm{PK}_{\mathrm{DO}}\right) \rightarrow \mathrm{CK}_{\mathrm{F}}
\end{gathered}
$$

The irreversible hash code and its secret key are attached with the data owner's cipher-text file and that will be transferred to the service provider. The secret key is maintained in separate table by the data owner.

The data owner periodically updates the access control list table and user_rekey table to retrieval of data. When the service provider is receiving a new request from the user, then he applies re-encryption procedure which is described above and transfer the content to the user.

After receives the content, the user applies the decryption to view the content. The decryption process has two levels. In the first level, the received content is decrypted using his re_key and Applies Hash_MAC algorithm for integrity verification. Then, the user applies the second level decryption using his secret key.

$$
\begin{gathered}
\text { User: } \mathrm{D}_{1}\left(\mathrm{RC}_{\mathrm{F}}, \mathrm{RK}_{\mathrm{DO}_{\rightarrow} \text { user }}\right) \rightarrow \mathrm{C}_{\mathrm{F}} \\
\text { User: } \mathrm{D}_{2}\left(\mathrm{C}_{\mathrm{F}}, \mathrm{SK}_{\mathrm{user}}\right) \rightarrow \mathrm{F}
\end{gathered}
$$

In this approach, it applies the asymmetric key encryption algorithm that is the data owner uses his public key to encrypt the data and user uses his secret key to decrypt the cipher-text. This approach has following salient features. This approach is simple to apply. Also, it enforces the selective authorization correctly. But, this approach is not suitable for the real time applications. Here, we are using asymmetric encryption algorithm for the protection. But the speed of the asymmetric algorithm is very slower than symmetric algorithm when large amount of data used.

\section{b. Multi Key Approach}

In the multi key approach, we use symmetric key encryption instead of asymmetric key algorithm. In the first level encryption, it generates the random key for each files of the data owner. Even though it generates the more keys for each file, there is no need to key distribution between the users and owner. We need only add a new column ekey to corresponding encrypted table. First, the data owner randomly chooses an encryption key randk $\mathrm{k}_{\mathrm{i}}$ for each file $\mathrm{F}_{\mathrm{i}}$ and encrypts the file to corresponding encrypted value $\mathrm{C}_{\mathrm{fi}}$ by using symmetric encryption algorithm. At the same time, the data owner uses same random key to generate the hash code which will be added with file. The data owner encrypts randk $\mathrm{k}_{\mathrm{i}}$ to the encrypted value $C_{\text {ranki }}$ in the $i^{\text {th }}$ row of the encryption key column.

$$
\begin{gathered}
\text { DO }: \text { E }\left(\operatorname{randk}_{\mathrm{i}}, \mathrm{F}_{\mathrm{i}}\right) \rightarrow \mathrm{C}_{\mathrm{fi}}: \text { CSP } \\
\text { DO: H_MAC }\left(\mathrm{randk}_{\mathrm{i}}, \mathrm{C}_{\mathrm{fi}}\right) \rightarrow \mathrm{H}_{\mathrm{fi}} \\
\text { DO: } \mathrm{E}\left(\mathrm{PK} \mathrm{DO}_{\mathrm{DO}}, \text { randk }_{\mathrm{i}}\right) \rightarrow \mathrm{C}_{\text {ranki }}: \text { CSP }
\end{gathered}
$$

Service provider stores $\mathrm{C}_{\text {ranki }}$ for later query and executes the remaining phases. In the second level encryption phase, the service provider performs the re-encryption of random key and sends it to the user.

$$
\text { CSP: } \mathrm{RE}\left(\mathrm{C}_{\text {ranki }}, \mathrm{rek}_{\mathrm{DO}_{\rightarrow} \text { user }}\right) \rightarrow \mathrm{rC}_{\text {ranki }}: \text { User }
$$

Data requester first uses his secret key $\mathrm{SK}_{\mathrm{user}}$ and decryption algorithm $\mathrm{D}$ to obtain the randk $\mathrm{k}_{\mathrm{i}}$ from $\mathrm{rC}_{\text {ranki }}$. Then user applies the standard decryption algorithm to get the plaintext using randk $\mathrm{k}_{\mathrm{i}}$.

$$
\begin{gathered}
\text { User: } \mathrm{D}\left(\mathrm{SK}_{\mathrm{user}}, \mathrm{rC}_{\text {ranki }}\right) \rightarrow \text { randk }_{\mathrm{i}} \\
\text { User: } \mathrm{SD}\left(\mathrm{randk}_{\mathrm{i}}, \mathrm{C}_{\mathrm{fi}}\right) \rightarrow \mathrm{F}_{\mathrm{i}}
\end{gathered}
$$

The multi key approach has the following features: 1) In this approach, the data requester or user need not derive any new keys in terms of catalog of tokens. 2) The user needs to remains only his secret key. 3) It makes the full use of both 
symmetric and asymmetric encryption algorithm to improve the speed of the cryptographic algorithms. 4) It also has the advantage of not much more key distribution between the owner and the user even the owner generates different encryption keys for each file.

\section{B. Second Level Encryption}

If the user $\mathrm{R}$ is willing to view the data owner file $\mathrm{F}$, then the user sends the request to service provider with his authentic certificate. This certificate contains the user id, requested file information. The service provider authenticates the user with his certificate. Then, the service provider checks the access control list table and verifies permission of the file with the user. It can be done with select query.

\section{SELECT Count $(*)$ from table_name where file $=F$ and user_id=R_id;}

This query retrieves the result as either 1 or 0 based on the permission of the file with the user. If it is 1 , then user can view the file. Then, the service provider retrieves the user's reencrypt key from the user_rekey that updated by REKG polynomial time algorithm. The service provider applies reencryption algorithm on the encrypted file $\mathrm{C}_{\mathrm{F}}$. Re-encryption is described in the figure 4.1.

Consider the plain text $\mathrm{F}$ is converted into cipher text $\mathrm{C}_{\mathrm{F}}$ which is stored in the server $\&$ the user sends the request to view this file. The re-key $\mathrm{RK}_{\mathrm{DO}->\mathrm{R}}$ is generated by the $\mathrm{REKG}$ algorithm. Then, the service provider applies the re-encryption algorithm to the file $C_{F}$. User's re-key and cipher-text has given as input and generates outputs as re-cipher-text.

$$
\text { SP: } \mathrm{RE}\left(\mathrm{RK}_{\mathrm{DO}_{\rightarrow} \text { user, }} \mathrm{C}_{\mathrm{F}}\right) \rightarrow \mathrm{RC}_{\mathrm{F}} \text { user }
$$

After receives the re-cipher-text, the user performs the standard decryption algorithm to retrieve the plaintext. This described as follows:

$$
\text { User: } \mathrm{D}\left(\mathrm{RC}_{\mathrm{F}}, \mathrm{SK}\right) \rightarrow \mathrm{F}
$$

\section{Application}

This framework is useful for large and hierarchical organizations. For the e.g. this framework is applied in the hospital management. In the hospital, various categories of the employees are available like specialist doctors, general ward doctors, duty doctors and nurses etc. The patient information is available in the hospital management. When the specialist doctor sends the patient report, only the specific doctors and nurses should view their patient reports. The access control list and proxy key list was updated in the respective databases. When the doctors request their patient information, they will send the patient id and requested information with his id. If he has the access limit, then server performs the re-encryption with the doctor's proxy key and sends to the doctor. Using his secret key, he may retrieve his patient's information. For the secure storage, the patient's information and files are stored in encrypted form.

\section{Access Control Tables}

Access control is an important mechanism in database management systems. In the cloud computing architecture, we explored selective encryption to enforce access control [11]. Recently, some of them explored selective encryption but those are inefficient. They adopt key distribution based on a user in which each user must derive all the authorized keys. Also, it does not support selective access to encrypted database which is encrypted by encryption key. To improve the selective authorization, we present re-encryption mechanism that provides flexible access control management.

\section{Access Matrix}

Access matrix is a conceptual model that represents access rights of file with the user. In this, access rights are represented in the form of matrix. Inside of the matrix, the access right values are represented as either ' 0 ' or ' 1 '. If the subject has the rights to access the file or object then it represented as ' 1 ' otherwise it is represented with ' 0 '. In the matrix, subject which is used to say the user is represented in row and the object is file which is represented in column. If the cell value of $A[s, o]$ is ' 1 ', then the user $s$ have the rights to read the file o. Access rights are also represented with Access Control List (ACLt) and Capabilities List (CAPs) [11]. In access control list, ACLt is related with tuple that which objects can access that tuple. In the capabilities list, CAPs represents subject which can access what are the tuple. For example, here we represent with four subjects and access rights with three files.

$$
\begin{array}{l|lll|} 
& F 1 & F 2 & F 3 \\
\text { Raj } & 1 & 1 & 0 \\
\text { Mani } & 0 & 1 & 0 \\
\text { Sekar } & 1 & 0 & 1 \\
\text { Siva } & 0 & 1 & 1
\end{array}
$$

Selective authorization for the access matrix is performed with following approaches:

1) One encryption key for each tuple: In this, for each file they have separate keys. It makes the user to remember many keys. It makes the key distribution complex.

2) One encryption key for all tuple: In this, the user using only a single data encryption key for all the files. Most of the systems are using this concept currently. It has the drawback that each user can access the whole delegated database. The key distribution is simple. But, it reduces the performance in order to filter the unauthorized users of the tuple.

But in our approach, the data owner should delegate some additional authorization table to the service provider disclosing any private information of the users. Those authorization tables are described as follows. The data owner should create at least two tables, 'user_rekey' and 'user_tuple'.

user_rekey (userid, name, rekey): this table contains the information about re-encryption keys with their user's information. It contains three fields. The first field is userid which is used to identify the user. It is the unique identifier to recognize the legitimate user. Each user has individual unique identifier. The second field gives the name of the user. The third field is rekey which gives the information of the user. For each legitimate user, they have separate re-encryption key. Using the standard SQL statement, the user or service provider can retrieve the re-encryption key. The retrieving statement is follows. 


\section{SELECT rekey FROM user_rekey WHERE userid=req_user_id}

User_tuple (userid, fileid): In our approach, each file has unique identifier for identifying a file. This table describes about access control lists of all the subjects. It contains the information that what are the files can accessed by the particular user. The information of accessing files by the user is retrieved from the table user_tuple using standard SQL statement.

\section{SELECT fid FROM user_tuple WHERE userid=req_user_id}

After the verification the legitimate user, we can easily retrieve the information of the file and re-encryption key for the legitimate user by the service provider. Both of these tables should be delegated to the service provider with secure channel.

\section{Dynamic ENVIRONMENT UPDATION Process}

In the cloud computing architecture, the data contents are shared with more number of users. Revoking an existing user or inserting a new user is great challenge in the shared architecture. When the user is relieving from the group, the data owner should download the content from the service provider and decrypt to obtain the plaintext. After that, generate the new encryption key and encrypt with new encryption key. Also, the owner should distribute the new encryption key to other users. It makes expensive cost for communication and computation for each revoking. But in our approach, revoking or inserting a user takes only less communication and computation cost. There are two different types of updating operations available in the dynamic environment. One is inserting or revoking the user from the group and another is inserting or deleting a resource. In our approach, they need not do more computation and also no need to derive any new key, when the users or resources are revoking.

\section{User Updating}

When we inserting a new user, the user should access the key distribution center and obtain their private and public key pair. Then, the owner will verify the user's identifier to authenticate the user. Here, the data owner only modifies the authorization tables for the user's updation. Then, the data owner generates the re-encryption key rek $\mathrm{DO}_{\rightarrow}$ user for the new user using re-encryption key algorithm. The owner inserts a tuple about the user with the corresponding accessing files and corresponding re-encryption key in their corresponding authorization tables using standard SQL statement.

Assume the new user is regu and his id is 1201, then the data owner generates the re-encryption key.

$$
\mathrm{REKG}\left(\mathrm{PK}_{\mathrm{DO}}, \mathrm{SK}_{\mathrm{DO}}, \mathrm{PK}_{\mathrm{regu}}\right) \rightarrow \mathrm{RK}_{\mathrm{DO}_{\rightarrow} \text { regu. }}
$$

Then the data owner updates the user information in both local and service provider's user_rekey table by using following SQL statement.

INSERT INTO user_rkey(userid, name, key) VALUES(1201,'regu', $\left.\mathrm{RK}_{\mathrm{DO}_{\rightarrow} \text { regu }}\right)$
When the user $u$ is relieving from the group, the data owner requests the service provider to delete the user tuple u from both authorization tables' user_rekey and user_tuple.

DELETE FROM user_tuple WHERE userid=1201 and

$$
\text { DELETE FROM user_rekey WHERE userid=1201 }
$$

\section{Resource updating:}

In our approach, consider that the files are as resources. When the owner wants to store a new file, he should encrypt the new file and send the encrypted file to the service provider without updating the policy that is no need to update the authorization tables. When the owner wants to delete the file, the data owner only needs to requests the service provider to delete all the tuple in the authorization table user_tuple.

\section{IMPLEMENTATION \& EVALUATION}

The first goal of our experiment is to demonstrate the $P K E$ approach, which applies the Re-encryption mechanism into the DaaS paradigm directly. We intend to show this is not practical because the decryption of asymmetric algorithm (RSA) spends much more time than that of two times decryption of symmetric algorithm (DES). When compared to the approaches proposed by $[9,10]$, our approach is better fit for the light user. This is because these methods require less computation for the DR in client. We use SQL server 2005 Express edition to store data on the server side and use the VS.NET 2005 as the integrated development environment, coding in C\# with Framework 2. We choose a symmetric algorithm AES and an asymmetric algorithm RSA. There is no standard provider re-encryption algorithm implemented, therefore we adopt RSA algorithm to imitate the re-encryption mechanism in our experiment evaluation, because they have the same work principle: public key for encryption and private key for decryption. However, they have an essential difference. The public and private key pairs of RSA belong to the same person, the public and private key pairs of server reencryption mechanism belong to different person, and the public key for the data owner and the private key for the data requester are all different. Using the asymmetric algorithm RSA to encrypt large data is more inefficient than that of symmetric algorithm AES, which couldn't be accepted by the online users. This characteristic of RSA is typically used by us to encrypt the data encryption key in our approaches and make more efficiency in the $D R$ than that of $[9,10]$ because their approaches need much more encryption and decryption operations in order to protect the confidentiality of the policy. For evaluation of the proposed system (SDS) performance we have compared the system with the recent approaches proposed in [20,21,22]. In [20], SeDaSC methodology encrypts a file with a single encryption key. SeDaSC generates two keys and one key is given to user and another key is stored in cryptographic server. By using these two keys security is provided. In [21], (SSD) proxy re-encryption algorithm based on heterogeneous cipher-text transformation and a user process protection method based on a virtual machine monitor, which provides support for the realization of system functions is proposed. In [22], (MDS) a hybrid solution for privacy preserving medical data sharing in cloud, where 
the technologies of statistical analysis and cryptography are innovatively combined together to support multiple paradigms of medical data sharing with multiple levels of privacy is proposed. The performance evaluation is done with the following parameters, Key generation time, file encryption and decryption time, file upload and download time. From figures 6.1,6.2,6.3 it is evident that, the proposed framework outperforms other recent approaches for secured data sharing in cloud computing and big data environment.

\section{SECURITY ANALYSIS}

In this section, we discuss and analyze the security features of our approach. In our approach, the first level encryption is performed by the data owner and second level encryption is performed by the service provider. This service provider re-encryption mechanism can provide the guaranteed privacy and also provide the flexible access control management. In this approach, only the legitimate users who having the proper secret key corresponding to re-encryption key can decrypt the cipher-text and get the original plaintext. Access control authorization tables are maintained by both the service provider and data owner. Service provider should know all the details of the access matrix and access control capabilities. Then only, the service provider can provide flexible access control correctly.

Guaranteed Integrity: In this approach, the cipher-text is given as input to the secure hash algorithm H_MAC and generates the hash code. That hash code enclosed with the cipher text. When the receiver receives the re_encrypted text, the receiver can verify the integrity by generating and comparing with new generated hash code. Hash code is irreversible code and even small change in the text will affect the hash code. Since, the receiver guarantees the cipher text is not modified by any other user.

Alternative approach: Instead of enclosing the hash code with cipher-text, the data owner creates a new table that contains the file and hash code information. The data owner only needs to update the integrity table when the new files to be uploaded. The receiver retrieves the hash code through the simple SQL query.

Guaranteed Privacy: in this approach, the encrypted cipher-text is delegated into the cloud service provider. Even if the re-encryption keys are available in the service provider, they cannot disclose the plaintext from the cipher-text. Only by the legitimate user's private key which is corresponding to re-encryption key can disclose the plaintext. In this approach, the sensitive data of the data owner is provided to the requested user by two level protections which provide more security for the content. The user can decrypt the cipher-text only after the second level protection.

Man in the middle attack: In the existing approach, the each cipher-text is re-encrypted with same re-encryption key. So, it generates the same re-cipher-text for the various ciphertexts. But, in this approach they are generating the random encryption keys. So it generates the different random re-encryption keys for the same user. It generates the various re-cipher-texts for the same cipher-text. So it makes the confusion and avoids the man in the middle attacks. Even if they have received the re-cipher-text, they cannot afford the original information from the re-cipher-text without having the legitimate user's secret key.

\section{CONCLUSION}

Since the cloud computing is providing everything as a service, the data contents of individual or small enterprises are delegated to the service provider. But, before transfer the data contents to the server, we should resolve all the security problems that are available in the DaaS paradigm. In this paper, we discussed the security issues related with database service paradigm and provide the efficient access control based re-encryption solution to secure the data from the inside and outside attackers. In this approach, we are providing two level protection using re-encryption mechanism. It reduces the communication cost and provides the flexible access control management. Our mechanism efficiently combines the reencryption algorithm with the access control policy of the data owner. To provide the flexible access control, it uses the access control authorization tables. Here, key generation is managed by the semi-trusted certified key distribution center. This framework provides confidentiality for the user. If the collusion occurs between the user and service provider, the user can disclose all the information which can encrypted by the user re-encryption. Since, we need to develop the efficient secret share based re-encryption mechanism for the above collusion problem.

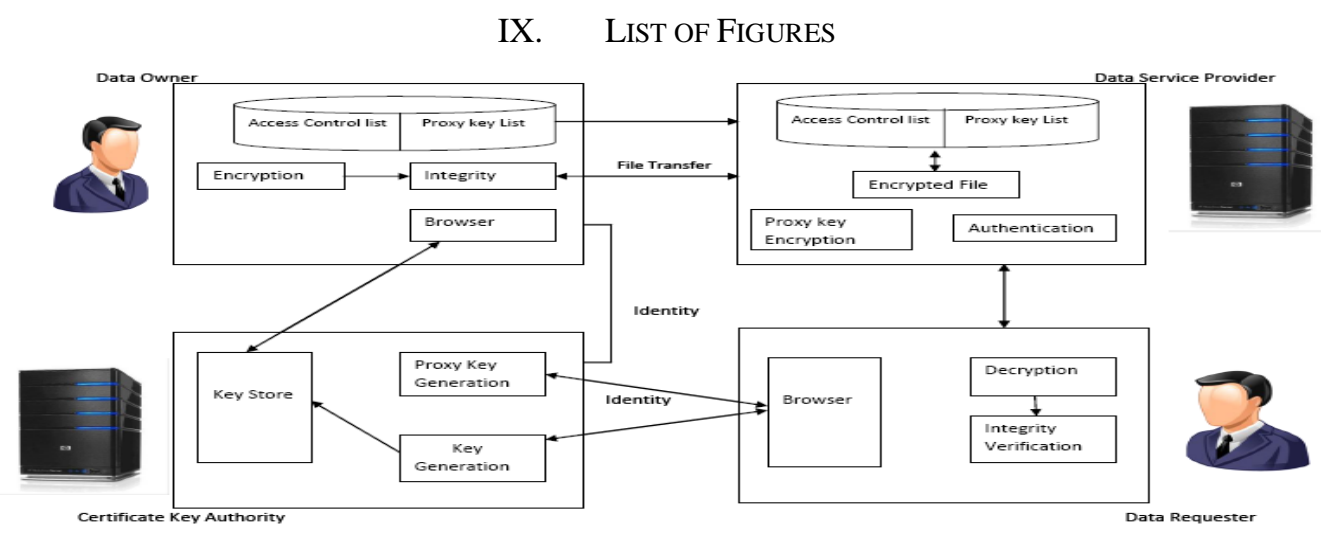

Fig. 3.1: System Model 


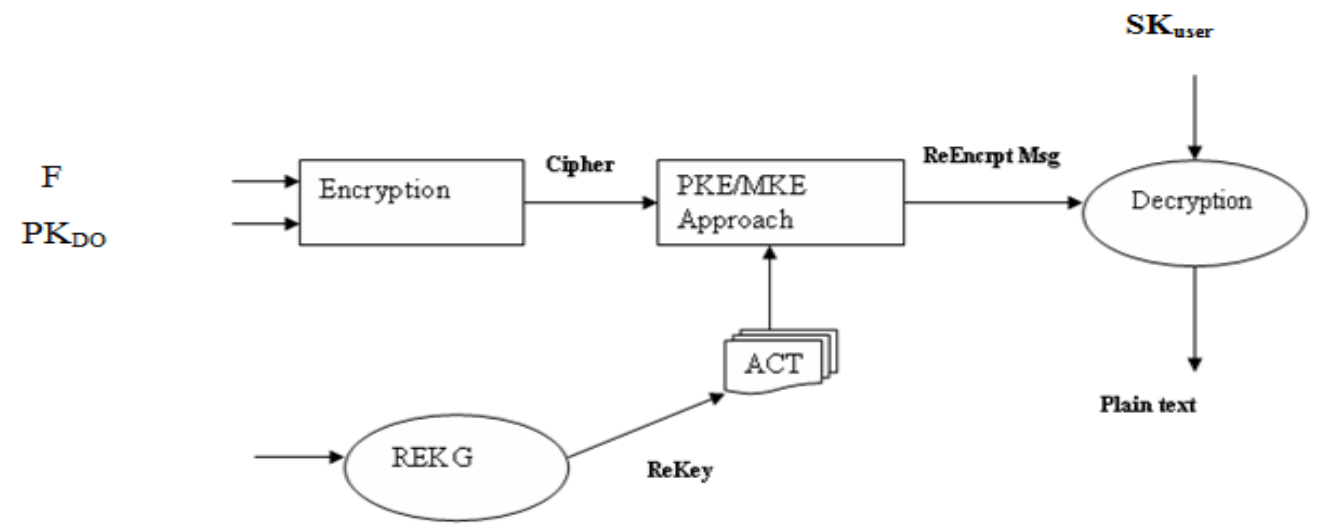

Fig. 4.1: Re-encryption Process

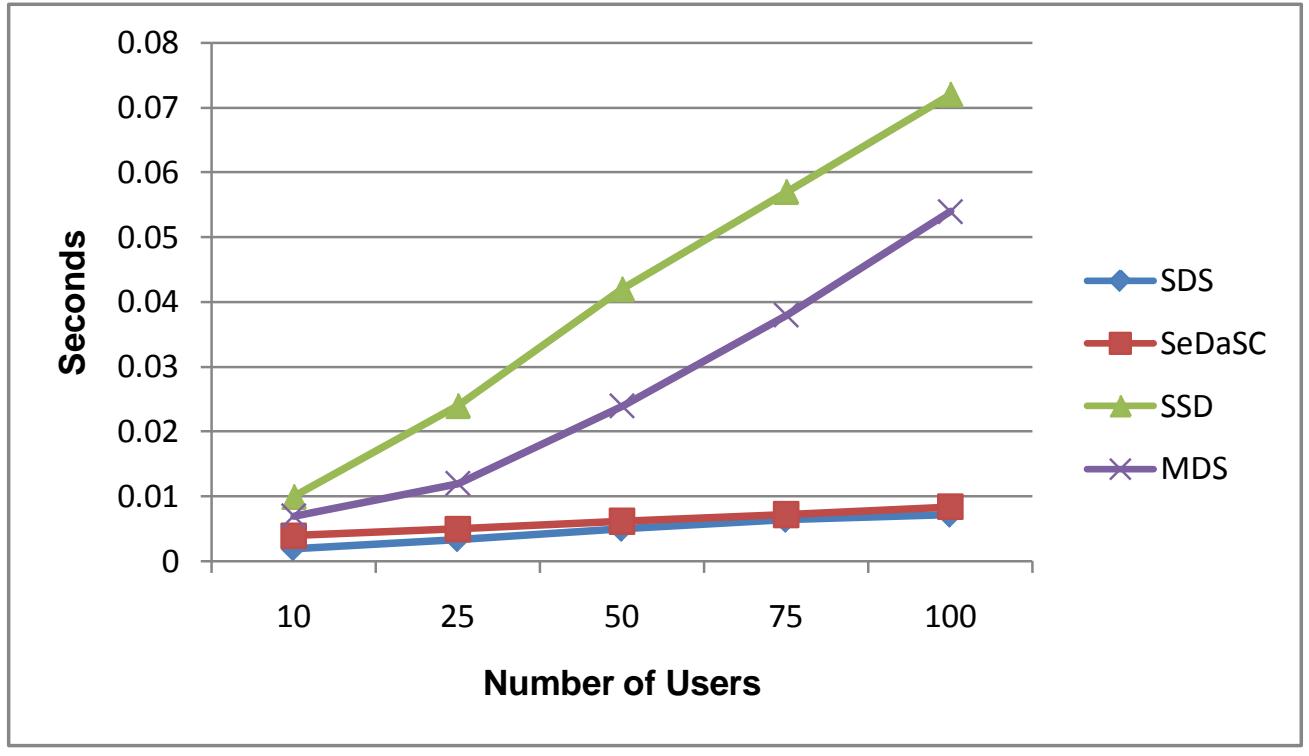

Fig. 6.1: Key Generation Time

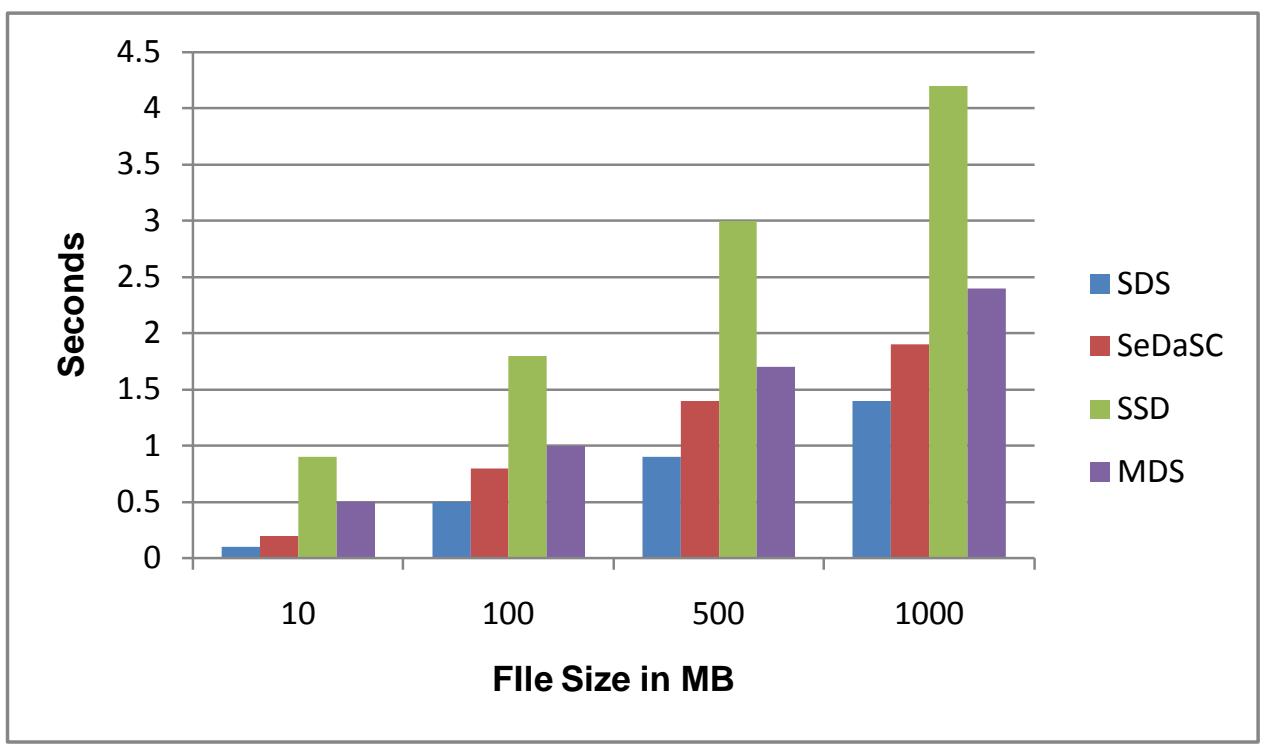

Fig. 6.2: File Encryption \& Decryption Time 


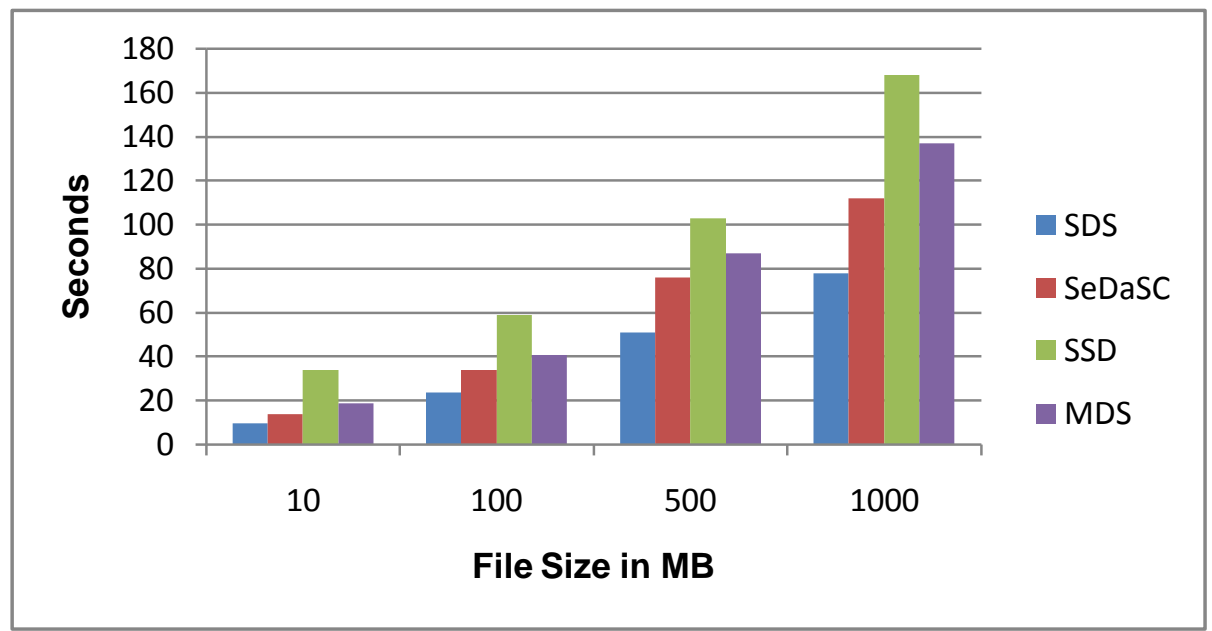

Fig. 6.3: File Uploading \& Downloading Time

\section{REFERENCES}

[1] D. Agrawal, A.E. Abbabi, F. Emekci and A. Metwally. "Data management as a service: Challenges and Opportunities”, Tech. rep., 2009.

[2] R. Agrawal, J. Kiernan, R. Srikant and Y. Xu, "Order preserving encryption for numeric Data”, ACM SIGMOD Conference(SIGMOD), Pp. 563-574, 2004.

[3] T. Allard, N. Anciaux, L. Bouganium, Y. L. Guo, L. Folgoc, B. Nguyen, P. Pucheral, I. Ray and S. Yin, "Secure personal data servers: a vision paper", Proceedings of the VLDB Endowment, Vol. 3, No. 1-2, Pp. 25-35, 2010.

[4] G. Ateniese, K. Fu, M. Green and S. Hohenberger, "Improved proxy re-encryption schemes with applications to secure distributed storage", Proceedings of the Annual Network and Distributed System Security Symposium, Pp. 83-107, 2005.

[5] L. Bouganim and P. Pucheral, "Chip-secured data access: confidential data on untrusted Servers”, VLDB Conference, Pp. 131-142, 2002.

[6] V. Ciriani, S. De Capitani di Vimercati, S. Foresti, S. Jajodia, S.Paraboschi and P. Samarati, "Fragmentation and encryption to enforce privacy in data storage", Proceedings of ESORICS, Pp. 171-186, 2007.

[7] C. Wang, S.S.M. Chow, Q. Wang, K. Ren and W. Lou, "PrivacyPreserving Public Auditing for Secure Cloud Storage”, Transactions on Computers, Vol. 62, No. 2, Pp. 362-375, 2013

[8] E. Damiani, S. De Capitani di Vimercati, S. Foresti, S. Jajodia, S. Paraboschi and P.Samarati, "Metadata management in outsourced encrypted databases", Lecture Notes in Computer Science, Secure Data Management, Pp. 16-32, 2007.

[9] S. De Capitani di Vimercati, S. Foresti, S. Jajodia, S. Paraboschi, G. Pelosi and P. Samarati, "Preserving confidentiality of security policies in data outsourcing", 7th ACM workshop on Privacy in the electronic society, Pp. 75-84, 2008.

[10] S. De Capitani di Vimercati, S. Foresti, S. Jajodia, S. Paraboschi and P. Samarati, "A data Outsourcing architecture combining cryptography and access control", Proceedings of the 1st Computer Security Architecture Workshop, Pp. 63-69, 2007.

[11] S. De Capitani di Vimercati, S. Foresti, S. Jajodia, S. Paraboschi and P. Samarati, "Over-Encryption: management of access control evolution on outsourced data", Proceedings of the 33rd VLDB Conference, Pp. 123-134, 2007.

[12] B. Iyer H. Hacigumus and S. Mehrotra, "Providing database as a service", Proceedings of 18th International Conference on Data Engineering, Pp. 29-38, 2002.

[13] H. Hacigumus, B. Iyer, S. Mehrotra, and C. Li, "Executing sql over encrypted data in the Database-serviceprovider model”, Proceedings of the ACM SIGMOD, Pp. 216-227, 2002.

[14] B. Libert and D. Vergnaud, "Tracing malicious proxies in proxy re-encryption", Lecture Notes in Computer Science, Pairing-Based Cryptography-Pairing, Pp.332-353, 2008.

[15] G. Miklau and D. Suciu, "Controlling access to published data using cryptography”, Proceedings of the $29^{\text {th }}$ VLDB conference, Pp. 898-909, 2003.
[16] M. Narasimha and G. Tsudik, "Dsac: integrity for outsourced databases with signature Aggregation and chaining”, Proceedings of the 14th ACM ICIKM, Pp. 235- 236, 2005.

[17] N. Shang, M. Nabeel, F. Paci and E.Bertino, "A privacy preserving approach to policy-Based content dissemination," in Proceedings of 26th ICDE Conference, Pp. 944-955, 2010.

[18] A. Velagapalli and M. Ramkumar, "Trustworthy tcb for dns servers", International Journal of Network Security, Vol. 14, No. 4, Pp. 187-205, 2012.

[19] A. Zych, M. Petkovi, and W. Jonker, "Efficient key management for cryptographically Enforced access control”, Computer Standards and Interfaces, Vol. 30, No. 6, Pp. 410-417, 2008.

[20] M. Ali, R. Dhamotharan, E. Khan, S. U. Khan, A.V. Vasilakos, K. Li, A.Y. Zomaya, "SeDaSC: Secure Data Sharing in Clouds", IEEE Systems Journal, 2015.

[21] Xinhua Dong, Ruixuan Li, Heng He, Wanwan Zhou, Zhengyuan Xue and Hao Wu, "Secure sensitive data sharing on a big data platform", in Tsinghua Science and Technology, Vol. 20, No. 1, Pp. 72-80, 2015.

[22] J.J. Yang, J.Q. Li and Y. Niu, “A hybrid solution for privacy preserving medical data sharing in the cloud environment", Future Generation Computer Systems, Pp. 74-86, 2015. 